\title{
TECNOLOGIA CRISTALIZADA E PRODUTIVIDADE TOTAL DOS FATORES ${ }^{1}$
}

Eliseu Alves ${ }^{2}$

Resumo - Conhecida a função de produção $y=f\left(x_{1}, x_{2}, \ldots, x_{s}\right)$, que indica o valor de y para cada valor do vetor de insumos, ou seja, o crescimento da produção somente ocorre em conseqüência de deslocamento ao longo da fronteira de produção e requer o uso de combinações diferentes de insumos, o que implica, portanto, novos dispêndios. Outra representação da estrutura de produção é dada por $y=f\left(x_{1}, x_{2}, \ldots, x_{s}, t\right)$, em que $\mathbf{t}$ não é um insumo e pertence aos números reais não-negativos. Agora, cada valor do vetor $x_{1}, x_{2}, \ldots, x_{s}$, produzirá uma quantidade diferente de $\mathrm{y}$, à medida que $\mathrm{t}$ varia, ou seja, é possível obter uma produção maior sem incorrer em custos. A questão que se coloca é a seguinte: $\mathrm{O}$ mundo real permite distinguir entre as duas representações acima, qual delas é a de melhor poder descritivo? A resposta é não, ou seja, o mundo real não permite distinguir tecnologia cristalizada da não-cristalizada, no sentido que será especificado. Para dirimir a questão da tecnologia cristalizada, não se fará, diretamente, uso da função de produção, mas sim das taxas de crescimento do produto e dos insumos.

Palavras-chaves: Função de produção, tecnologia, insumos.

\section{Introdução}

Caso não haja novo insumo e novo conhecimento de como usar os insumos conhecidos; então o crescimento da produção somente poderá ocorrer em conseqüência de deslocamento ao longo da fronteira de produção. Desse modo, toda variação da produção será atribuída à variação nas quantidades de insumos usados, ou o conhecimento das quantidades dos insumos fornecerá, imediatamente, o conhecimento das quantidades de produtos. Toda a informação sobre a produção cristalizará nos insumos;

${ }^{1}$ Recebido em 01/10/2004 Aceito em 29/10/2004

${ }^{2}$ Eliseu Alves é pesquisador da Embrapa. 
portanto, todo aumento da produção incorrerá em um valor diferente para o custo.

Aprendemos, agora, algo novo sobre determinado insumo, sem qualquer alteração de suas propriedades físicas, e este algo novo traz, para o mesmo nível de insumos, um acréscimo de produção. Quebrou-se a correspondência entre as variações nas quantidades de insumos e as variações nas quantidades de produtos, com o mesmo vetor de insumos produz-se um novo vetor de produtos. Estamos, agora, frente a frente com as duas visões do processo produtivo. A primeira diz que a função de produção se deslocou para cima; assim, o aumento da produção não está apenas cristalizado nos insumos; a segunda diz que houve erro na mensuração dos insumos. Por ela, o novo conhecimento dará origem a um novo insumo. A primeira visão é representada pela função $y=f\left(x_{1}, x_{2}, \ldots, x_{s}, t\right)$; a segunda diz que tem de corrigir o insumo que sofreu o influxo do novo conhecimento, multiplicando-o por um número positivo, sendo a nova representação obtida por $y=f\left(x_{1}, x_{2}, \ldots, \alpha^{*} x_{k}, \ldots, x_{s}\right)$, em que o insumo modificado $x_{k}$ é multiplicado por $\alpha>1$, o que equivale a introduzir o novo insumo $x^{\prime}=\alpha^{*} x_{k}$ e $y=f\left(x_{1}, x_{2}, \ldots, x^{\prime}, \ldots, x_{s}\right)$. aos dados.

O leitor atento deverá está indagando como muda a função de produção, no caso de progresso tecnológico cristalizado. A resposta é que ela não muda. Em um horizonte de cem anos, no qual se dispõe da lista de insumos para este período, e tem-se uma função que transforma os insumos em produtos. Em muitos anos, vários insumos não comparecerão, serão registrados zeros. Os insumos sobre os quais se adquiram novos conhecimentos serão introduzidos com outros símbolos. Na data da introdução, o novo símbolo aparecerá e o velho símbolo será zero, e novo símbolo será zero nos anos anteriores à introdução.

Os que se opõem ao modelo cristalizado alegam que sempre restará um resíduo, não explicado pela função de produção, o qual é atribuído ao 
progresso tecnológico não-cristalizado. A tese, desenvolvida por Jorgenson, é que os dados da economia não permitem testar o modelo de produção de tecnologia cristalizada diante do de tecnologia não-cristalizada. Quem quiser aprofundar nesse assunto e nas controvérsias do passado, ver Jorgenson e Griliches, 1967, Jorgenson, 1995.

O trabalho formalizará a discussão em curso, sem usar a função de produção. Para cada índice de progresso tecnológico cristalizado, terse-á o correspondente índice de progresso tecnológico não-cristalizado. Por isto, os dados não discriminam as duas representações de tecnologias, no sentido das taxas de crescimento do produto e dos insumos.

\subsection{Preliminares}

É necessário conhecer a fórmula do crescimento do capital para desenvolver o resultado que está no final do trabalho. A seção não precisa ser lida numa primeira leitura; deve-se, contudo, entender a equação (2).

O capital deprecia-se a uma taxa instantânea positiva $\mathrm{d}>0$. Por isto, parte do investimento feito visa substituir o capital depreciado. A necessidade de investimento, para essa finalidade, é dada por I(t), na data $t$. $I(t)=e^{d t} i(t) d>0$ e $t>0$. Logo, $\mathrm{t}>0, \mathrm{I}(\mathrm{t})>\mathrm{i}(\mathrm{t})$, porque $e^{d t}>1$. A diferença $I(t)-i(t)=i(t)\left(e^{d t}-1\right)$ equivale ao investimento feito para substituir o capital que foi depreciado. Note-se que, ao se decidir investir em $\mathrm{i}(\mathrm{t})$, o investimento necessário tem de ser maior e igual a $\mathrm{I}(\mathrm{t})$. Está-se ainda falando da depreciação instantânea do capital, que o investimento $\mathrm{i}(\mathrm{t})$ cristalizou, por hipótese, igual a $\mathrm{i}(\mathrm{t})$. Derivando-se $\dot{K}(t)=-d K(t)+i(t)$, e dividindo-se por $\mathrm{I}(\mathrm{t})$, ter-se-á,

$$
\frac{i(t)}{I(t)}=d+\frac{\dot{i}(t)}{i(t)}
$$


Assim, a taxa de crescimento do investimento, $\mathrm{I}(\mathrm{t})$, é igual à taxa de depreciação mais a taxa de crescimento do investimento feito, $i(t)$.

Seja $\mathrm{K}(\mathrm{t})$ o capital existente em t. Ele é o que sobrou da depreciação instantânea de $\mathrm{k}(\mathrm{t})$. Logo, $K(t)=e^{-d t} k(t) \quad(* *)$. Como $k(t)=\int_{\infty}^{t} e^{d s} i(s) d s\left(^{*}\right), \quad$ substituindo-se na fórmula ao lado, virá $K(t)=e^{-d t} \int_{\infty}^{t} e^{d s} i(s) d s$. Derivando-se em $(* *), \mathrm{K}(\mathrm{t})$ em relação a $\mathrm{t}$, tem-se $\dot{K}(t)=-d e^{-d t} k(t)+e^{d t} \dot{k}(t)$. Por $(* *), \dot{K}(t)=-d K(t)+e^{-d t} \dot{k}(t)$. Derivando-se $\mathrm{k}(\mathrm{t})$ em $(*)$, e substituindo-se na fórmula ao lado, $\dot{K}(t)=-d K(t)+i(t)$. Dividindo-se por $\mathrm{K}(\mathrm{t})$, obter-se-ão:

$$
\frac{\dot{K}(t)}{K(t)}=-d+\frac{i(t)}{K(t)}=-d+e^{d t} \frac{i(t)}{k(t)}
$$

$$
\frac{\dot{K}(t)}{K(t)}=-d+\frac{i(t)}{K(t)}=-d+\frac{I(t)}{\int_{-\infty}^{t} e^{d s} i(s) d s}
$$

O lado direito de (2) foi obtido pela substituição de $\mathrm{K}(\mathrm{t})$ pela sua expressão em (**), e (2) dá a taxa de crescimento do capital. Deixando-se t tender para o infinito e aplicando-se a regra de L'Hospital na segunda parcela do lado direito de (2), obtém-se

$$
\lim _{t \rightarrow \infty} \frac{\dot{K}(t)}{K(t)}=\frac{\dot{i}(t)}{i(t)}
$$

Assim, no longo prazo, o capital cresce à mesma taxa do investimento. 


\subsection{Crescimento da produtividade total}

Em uma economia que usa dois fatores de produção, $\mathrm{K}$ e L, e produz um bem de consumo, representado por $\mathrm{C}$, e acréscimo ao capital, representado por I, os preços são representados por $p e \pi$, e as variáveis são funções diferenciáveis do tempo, t. Por definição, a produtividade total será dada por

$$
P=\frac{p_{C} C+p_{I} I}{\pi_{K} K+\pi_{L} L}
$$

Definida a participação do investimento, de I, no valor do produto, $p_{C} C+p_{I} I, S_{I}^{P}=p_{I} I /\left(p_{C} C+p_{I} I\right)$. A participação de C corresponde a $S_{C}^{P}=p_{C} C /\left(p_{C} C+p_{I} I\right)$; e a participação do capital, K, no custo total corresponde a $S_{K}^{i n}=\pi_{K} K /\left(\pi_{K} K+\pi_{L} L\right)$. Finalmente, $S_{L}^{i n}=\pi_{L} L /\left(\pi_{K} K+\pi_{L} L\right)$, participação de L no custo total.

Derivando-se (3) em relação a t, dividindo-se o resultado por $\mathrm{P}$, e levando-se em consideração as definições anteriores, depois de manipulações simples virá:

$$
\frac{\dot{P}}{P}=\left(S_{I}^{P} \frac{\dot{I}}{I}+S_{C}^{P} \frac{\dot{C}}{C}\right)-\left(S_{K}^{\text {in }} \frac{\dot{K}}{K}+S_{L}^{i n} \frac{\dot{L}}{L}\right)
$$

O lado esquerdo de (4) é o crescimento da produtividade total. No lado direito, ela é dividida em duas parcelas, que estão entre parêntesis. A primeira delas equivale ao crescimento do produto, $p_{C} C+p_{I} I$; a Segunda, ao crescimento do custo, $\pi_{K} K+\pi_{L} L$. 


\section{Definição 1}

A tecnologia será cristalizada, se $\frac{\dot{P}}{P}=0$. Pela hipótese pela qual toda tecnologia é cristalizada, se $\frac{\dot{P}}{P}>0$, então, não se corrigiram adequadamente os insumos. Se $\frac{\dot{P}}{P}>0$, pode-se afirmar que a tecnologia é cristalizada? Nota-se que a definição não afirma "se e somente se". Mais abaixo, será demonstrado como isto pode ocorrer, equação (6) e considerações que lhe seguem.

Em palavras simples, se afirmar que a tecnologia será cristalizada, quando as taxas de crescimento do produto e do custo total forem iguais; e os preços forem mantidos constantes, isto não incomodara, porque estaremos lidando com acréscimos infinitesimais de t. Na prática, quando se trabalha com uma seqüência de anos, este é um problema complicado, que exige muita habilidade e paciência para resolvê-lo.

\subsection{Modelo simplificado de tecnologia cristalizada e não cristalizada}

A correção incidirá tão-somente no capital para simplificar as fórmulas. Corrige-se o investimento $\mathrm{i}(\mathrm{t})$, aplicando-se-lhe o índice $1 / \mathrm{Q}(\mathrm{t})$. Abandonar-se-á a notação $\mathrm{Q}(\mathrm{t})$, mas o leitor deverá lembrar-se de que todas as variáveis são funções de $t$, sendo os preços constantes. Corrige-se I no correr do tempo, de modo que seja medido em unidades comparáveis. Pela integral (*), que liga k e i, segue-se que k também é corrigido e, portanto, K. A correção é feita pela fórmula $I^{*}=(1 / Q) I$. , que equivale a $I=I^{*} Q$. Ao derivá-la em relação a t, depois de rearranjar os termos, obter-se-á, 
$-\frac{\dot{Q}}{Q}=\frac{\dot{I}^{*}}{I^{*}}-\frac{\dot{I}}{I}$

$(* * * *)$

Ora, $-\frac{\dot{Q}}{Q}$ é a taxa de crescimento do índice cristalizado de tecnologia, 1/Q. Pode-se interpretar 1/Q como um índice de qualidade ou, melhor ainda, como uma medida da tecnologia incrustada em I. Aliás, é o que a medida de qualidade faz.

Pela hipótese de que toda a tecnologia está incrustada nos insumos, o crescimento dos insumos esgotará a produção, se I e L forem adequadamente medidos. Por hipótese, para simplificar as fórmulas, L será corretamente medido, ao longo do tempo.

$\frac{\dot{P}^{*}}{P^{*}}=0=\left(S_{I}^{P} \frac{\dot{I^{*}}}{I^{*}}+S_{C}^{P} \frac{\dot{C}}{C}\right)-\left(S_{K}^{\text {in }} \frac{\dot{K}^{*}}{K^{*}}+S_{L}^{i n} \frac{\dot{L}}{L}\right)$

No modelo simplificado, pode-se admitir que I e K estejam crescendo à mesma taxa. No longo prazo, por (***), é claro que isto ocorre. A igualdade (5) é obtida de (4), em que I e K são corrigidos nos lugares de I e K.

Em (5), será adicionado a ambos os membros (os dois da direita) o membro da direita de (4), depois de algumas manipulações simples, 


$$
\begin{aligned}
& \left(S_{I}^{P} \frac{\dot{I}}{I}+S_{C}^{P} \frac{\dot{C}}{C}\right)-\left(S_{K}^{\text {in }} \frac{\dot{K}}{K}+S_{L}^{\text {in }} \frac{\dot{L}}{L}\right)=-S_{I}^{p}\left(I^{*} / I^{*}-\dot{I} / I\right)+S_{k}^{\text {in }}\left(\dot{K}^{*} / K^{*}-\dot{K} / K\right) \text { ou, } \\
& \frac{\dot{P}}{P}=-S_{I}^{p}\left(I^{*} / I^{*}-\dot{I} / I\right)+S_{k}^{\text {in }}\left(\dot{K}^{*} / K^{*}-\dot{K} / K\right)
\end{aligned}
$$

Obter-se-á (3a), porque o primeiro membro da igualdade acima, por (4), será igual a $\frac{\dot{P}}{P}$. Lembrando-se que K e I crescem, por hipótese, à mesma taxa, ter-se-á :

$$
\frac{\dot{P}}{P}=\left(S_{k}^{i n}-S_{I}^{p}\right)\left(\dot{I}^{*} / I^{*}-\dot{I} / I\right)
$$

Por (****) acima,

$$
\frac{\dot{P}}{P}=\left(S_{k}^{i n}-S_{I}^{p}\right)\left(-\frac{\dot{Q}}{Q}\right)
$$

Em (6), o fator à direita é taxa de crescimento da tecnologia cristalizada.

Para que a taxa de crescimento, $\frac{\dot{P}}{P}$, seja diferente de zero, é necessário 
que $S_{k}^{i n} \neq S_{I}^{p}$ e $\frac{\dot{Q}}{Q} \neq 0$. Mas, quando $\frac{\dot{P}}{P}>0$, se for conhecido, determina-se (6), imediatamente, $-\frac{\dot{Q}}{Q}$ e vice-versa. Assim, por (6), somente se terá liberdade para estimar $\frac{\dot{P}}{P}$ ou $\frac{\dot{Q}}{Q}$. Estimado um deles, imediatamente, obter-se-á o outro, e vice-versa. Assim, os dados não terão a capacidade de discriminar tecnologia cristalizada, representada pela taxa, e não-cristalizada, representada por .

\subsection{Relação investimento bruto - capital: modelo não-cristalizado}

Por definição, o produto, $\mathrm{O}$, é dado por $O=p_{I} I+p_{C} C$. Derivando-se em relação a te dividindo-se por t, obtém-se:

$$
\frac{\dot{O}}{O}=S_{I}^{p} \frac{\dot{I}}{I}+S_{C}^{p} \frac{\dot{C}}{C}
$$

Por 2, $\frac{\dot{K}(t)}{K(t)}=-d+\frac{i}{K}$. O primeiro membro da direita de (4) é $\dot{O} / O$ e em (4), substituindo-se $\dot{K} / K$ por $-d+i / K$, terá:

$$
\frac{\dot{O}}{O}=\frac{\dot{P}}{P}+S_{K}^{\text {in }} \frac{i}{K}-S_{K}^{i n} d+S_{L}^{i n} \frac{\dot{L}}{L}
$$


Resolvendo-se para ,

$$
\frac{i}{K}=\frac{\frac{\dot{O}}{O}-\frac{\dot{P}}{P}+S_{K}^{i n} d-S_{L}^{i n} \frac{\dot{L}}{L}}{S_{k}^{i n}}
$$

A equação (7) permite calcular a relação investimento bruto - capital para a tecnologia não-cristalizada, a exemplo de Jorgenson 1995, vol. 1, capítulo 2, 41 -42). Tomemos os seguintes dados da linha correspondente ao ano 1959, da Tabela 2.1, Jorgenson, 1995, p.35:

$S_{K}^{i n}=1-S_{L}^{i n}=0,35287, \quad S_{L}^{i n}=0,64713 \dot{L} / L=0,00594, \quad S_{I}^{p}=0,16665$.

Admitidos os seguintes valores para $\dot{O} / O=0,03 \dot{P} / P=0,25 \quad d=0,02$ e substituindo-os na fórmula (7), ter-se-á $i / K=0,028276$. E se tivéssemos de realizar o mesmo cálculo para a tecnologia cristalizada?

Na fórmula (7), ao substituir $P$ / $P$ pela expressão da fórmula 6 , ter-seá:

$$
\frac{i}{K}=\frac{\frac{\dot{O}}{O}+\left(S_{k}^{i n}-S_{I}^{p}\right)\left(\frac{\dot{Q}}{Q}\right)+S_{K}^{i n} d-S_{L}^{i n} \frac{\dot{L}}{L}}{S_{k}^{i n}}
$$


Comparando-se 8 com 7, o resultado terá de ser o mesmo. Para verificar, ao determinar $\dot{Q} / Q$, em (6), considerando-se as informações acima, encontrar-se-á $\dot{Q} / Q=0,1342$. Fazendo-se as substituições em (8), encontrar-se-á o mesmo valor para $I / K$, como teria que ser.

\subsection{Relação geral entre $\dot{P} / P$ e $\dot{Q} / Q$}

A base do desenvolvimento é a fórmula (3a), que será repetida para facilitar o acompanhamento. Note-se que se relaxou a hipótese de igualdade das taxas de crescimento.

$$
\begin{aligned}
& \frac{\dot{P}}{P}=-S_{I}^{p}\left(I^{*} / I^{*}-\dot{I} / I\right)+S_{k}^{i n}\left(\dot{K^{*}} / K^{*}-\dot{K} / K\right) \text {. Por }(* * *) \text { e como } \\
& -\frac{\dot{Q}}{Q}=\frac{\dot{I}^{*}}{I^{*}}-\frac{\dot{I}}{I}, \text { segue-se que }
\end{aligned}
$$$$
\frac{\dot{P}}{P}=S_{I}^{p}(\dot{Q} / Q)+S_{k}^{i n}\left(\dot{K}^{*} / K^{*}-\dot{K} / K\right)
$$

Por 2(a), tem-se $\frac{\dot{K}(t)}{K(t)}=-d+\frac{I(t)}{\int_{-\infty}^{t} e^{d s} i(s) d s}$ 
Se quiser obter $K^{*}(t) / K^{*}(t)$, tem-se, em (10), que dividir I (t) por $\mathrm{Q}(\mathrm{t})$ e i(s) por $\mathrm{Q}(\mathrm{s})$. Então,

$$
\frac{\dot{K}^{*}(t)}{K^{*}(t)}=-d+\frac{I(t)}{Q(t) \int_{-\infty}^{t} e^{d s}(i(s) / Q(s)) d s}
$$

Ao substituir (10) e (11) em (9), obter-se-á o resultado que se buscava.

$$
\frac{P(t)}{P(t)}=S_{I}^{p}(\dot{Q} / Q)+S_{k}^{i n}\left(\frac{I(t)}{Q(t) \int_{-\infty}^{t} e^{d s}(i(s) / Q(s)) d s}-\frac{I(t)}{\int_{-\infty}^{t} e^{d s} i(s) d s}\right)
$$

Embora complexa, a relação (12) demonstra que, obtido $\dot{Q} / Q$, derivase $\dot{P} / P$ e vice-versa. Novamente, os dados não discriminam tecnologia cristalizada e não-cristalizada.

\section{Considerações finais}

Na pesquisa aplicada, o usual é estimar P (produtividade total dos fatores) e regredir o resultado sobre determinadas variáveis, ou ainda, $\dot{P} / P$, variável dependente de alguma regressão que pretende explicar sua variação.

Aceita a hipótese da tecnologia cristalizada, $\dot{P} / P \neq 0$ significa que não se corrigiram corretamente os insumos. Assim, a busca das variáveis explicativas deve recair sobre aquelas que poderiam ter sido utilizadas 
na correção da série de insumos, de acordo com o preceito que reza que a série deva estar assentada numa base comparável. Assim, justifica-se educação como variável dependente, porque ela corrigiria o fator trabalho, gastos em pesquisa e assistência técnica, por duas razões: corrigiria muitos insumos e responderia pelo que não foi explicado pela tecnologia cristalizada; o índice pluviométrico corrigiria, em parte, a terra; potência dos tratores para considerar o progresso tecnológico de natureza mecânica.

Na literatura sobre produtividade total dos fatores (PTF), que propõe estimar P, não é usual se valer da hipótese da tecnologia cristalizada para escolher as variáveis exógenas da regressão; por isto, não se tem uma explicação convincente da escolha feita. Um trabalho recente é o de Vicente, Anefalos e Caser, 1993.

Assim, a contribuição de Jorgenson, além de elucidar os conceitos de tecnologia cristalizada e não-cristalizada e de ensinar a corrigir os insumos, é muito valiosa como guia à escolha das variáveis exógenas das regressões que propõem explicar a produtividade total dos fatores.

\section{Referências bibliográficas}

JORGENSON, D.; GRILICHES, Z. The explanation of productivity change. Review of Economic Studies, 34 (3), 99 (July), p. 249-280, 1967.

JORGENSON, D. Productivity: Postwar U.S. Economic Growth, Cambridge, Massachusetts, 1995.

JORGENSON, D. Productivity: international comparisons of economic growth. Cambridge, Massachusetts, 1995.

VICENTE, J. R.; ANEFAlOS, L. C.; CASER, D. V. Influência de capital humano, insumos modernos e recursos naturais na produtividade agrícola. Em Helfand, Steven M. e Rezende, Gervásio Castro 


\section{(organizadores) Região e espaço no desenvolvimento agrícola bra-} sileiro, p. 265-295, IPEA, Rio de Janeiro, 2003.

\footnotetext{
Abstract - Suppose that the production, $y=f\left(x_{1}, x_{2}, \ldots, x_{s}\right)$, is known. It says that we are able to know for each value of the input vector, $x_{1}, x_{2}, \ldots, x_{s}$, the correspondent value of $y$. Or yet production growth occurs as consequence of movement along the production frontier, and it requires a different combination of inputs, and consequently, a higher or a smaller expenditure. Another representation of the production structure is, $y=f\left(x_{1}, x_{2}, \ldots, x_{s}, t\right)$, where $\mathbf{t}$ is non negative real number. Now each set of $x_{1}, x_{2}, \ldots, x_{s}$ gives a different $\mathrm{y}$ as $\mathrm{t}$ varies. Or, it is possible to achieve a higher level of production with no additional cost. A question comes to mind: can the real world (or the data) say which one of the two representations has a better descriptive power? The answer is no. Or yet, the real world cannot distinguish between embodied and disembodied technology in a sense that will be introduced below. We will not use directly the concept of production function to establish the model appropriate to test embodied technology against the disembodied one. In its place, the rates of growth of products and inputs are instrumental.

Keywords: Production function, technology, inputs.
} 